\title{
ACCOUPLEMENT, PERIODE D'INCUBATION ET PREMIERS STADES LARVAIRES DE BRACHYPTERA BRAUERI ET DE PERLODES MICROCEPHALUS (PLECOPTERA)
}

\author{
par C. BERTHELEMY1
}

\begin{abstract}
Le mâle de Brachyptera braueri introduit d'abord son épiprocte dans le vagin. Après une longue phase de stimulation, il dépose un spermatophore externe. Chez les Brachyptera, il existe une corrélation interspécifique entre la longueur des filaments de l'épiprocte et du flagelle du paraprocte droit des mâles et la longueur du canal du réceptacle séminal des femelles. Les œufs de B. braueri éclosent au bout de trois à sept semaines. Ceux de Perlodes microcephalus éclosent soit deux à cinq mois après la ponte, soit un an après. La chétotaxie des prcmiers stades larvaires des deux espèces est décrite et figurée en détail. Les transformations évolutives du stade $I$ et de l'adulte montrent un curieux parallélisme, à la fois pour les cerques des Nemouroidea et les pièces buccales des Subulipalpia.
\end{abstract}

\section{Mating, incubation period of the eggs, and first larval stages of Brachyptera braueri and Perlodes microcephalus (Plecoptera).}

The male Brachyptera braueri first introduces his epiproct into the vagina and after a long phase of stimulation, he deposits a spermatophore externally. In the genus Brachyptera, there is an interspecific correlation between the length of the filaments of the epiproct and the flagellum of the right paraproct of the males and the length of the canal of the seminal receptacle in the females. The eggs of $B$. braueri hatch at the end of 3-7 weeks, whilst those of Perlodes microccphalus hatch either 2.5 months after oviposition or one year after. The chaetotaxy of first-instar larvae of both species is described and illustrated in detail. The evolutional transformations of the first larval stage and the adult show an interesting parallelism, together with the cerci of the Nemouroidea and the buccal parts of the Subulipalpia.

\section{1. - BRACHYPTERA BRAUERI (KLAPALEK, 1900)}

\subsection{Organes génitaux}

Les organes génitaux des Brachyptera ont été bien étudiés par Zwick (1973). Ceux de $B$. braueri avaient déjà été décrits par Klapálek (1896, sub nom. Taeniopteryx trifasciata) et les caractères propres à cette espèce sont précisés dans plusieurs faunes (Despax 1951, Brinck 1952, Illies 1955, Hoffmann 1960, Kis 1974). Aussi me bornerai-

1. Laboratoire d'Hydrobiologie (ERA 702), Université Paul-Sabatier, 118, route de Narbonne, 31077 Toulouse Cedex. 
je aux données nécessaires pour exposer les modalités de l'accouplement.

\subsubsection{Males}

Le sclérite basal de l'épiprocte porte un bulbe arrondi (Berthélemy 1971, fig. 1 et 4, B., Zwick 1973, fig. 50 A) d'où se détache un procès. En arrière, une gaîne membraneuse contient trois filaments sclérifiés (fig. 23 à 26). Ces filaments sont longs chez B. braueri, plus courts chez B. risi (Morton, 1896) et surtout chez B. monilicornis (Pictet, 1841 ) et $B$. seticornis (Klapálek, 1902). Leur partie proximale est enroulée et située dans la moitié gauche du bulbe. Leur extrémité est munie d'aspérités dont la pointe est dirigée vers la base (fig. 15 à 18).

Les cerques présentent un appendice basal dorsal (Berthélemy 1971, fig. 1 et 4 , A.C.).

Les paraproctes portent des formations dissymétriques (fig. 1 et 2). Chez $B$. braueri, le flagelle du paraprocte droit est long et muni d'une frange hélicoïdale, comme chez B. auberti Consiglio, 1957 (Zwick 1973, fig. $50 \mathrm{G}$ ). Cette frange est moins régulière chez les autres Brachyptera.

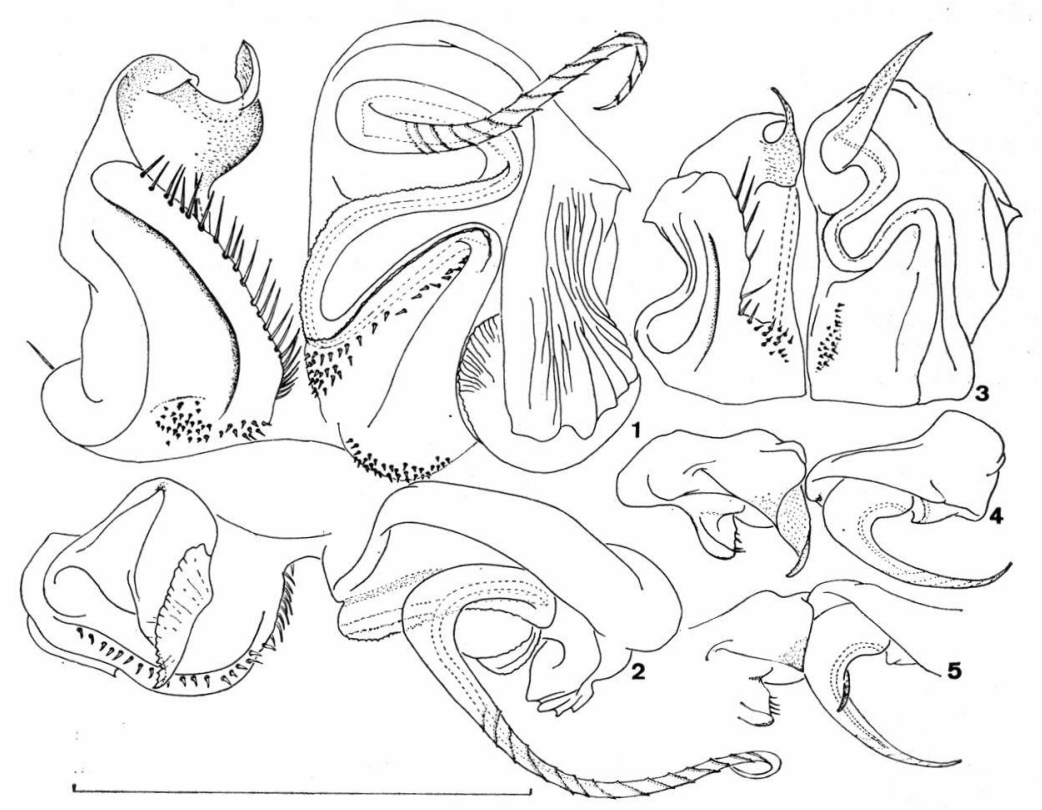

Fig. 1. - Formations paraproctales de Brachyptera braueri, vue ventro-postérieure. FIG. 2. - Id., vue dorso-postérieure. FIG. 3. - Formations paraproctales de B. monilicornis, vue ventro-postérieure. FIG. 4. - Id., vue dorso-postérieure. Fig. 5. - Id., flagelle du paraprocte droit engagé dans le prolongement membraneux du paraprocte gauche. Le trait horizontal correspond à $0,5 \mathrm{~mm}$. 
Les figures des formations paraproctales de B. monilicornis (fig. 3 à 5) complètent, pour les espèces françaises, celles que j'ai publiées précédemment (Berthélemy 1971, fig. 10, 11 et 14 à 17).

\subsubsection{Femelles}

Le bord antérieur de l'orifice génital constitue un bon critère de détermination (fig. 6 à 9). Les bords latéraux sont formés par des bourrelets rétractables, homologues des accessoires membraneux des Taeniopteryx décrits par Brinck (1956).

Le vagin est ceinturé par des muscles transversaux (fig. 20). Sa paroi antérieure présente des plis dorso-ventraux en partie visibles par l'orifice vulvaire. Sa paroi dorsale est munie d'une sclérification médiane chez B. risi, B. beali (Navás, 1923) et B. seticornis (fig. 21 et 22).

Les bourrelets vulvaires et les faces latérales du vagin sont couverts de mamelons ( $B$. braueri et $B$. monilicornis, fig. 11 et 12 ), de digitations arrondies (B. seticornis, fig. 14 ) ou aiguës (B. risi, fig. 13, et $B$. beali). Cette ornementation fait place à des microtriches allongés à l'entrée du canal du réceptacle séminal, qui débouche à l'arrière du vagin. La face interne du canal est nue chez $B$. braueri et $B$. monilicornis, couverte de microtriches sur le premier quart chez $B$. seticornis et sur toute sa longueur, jusqu'au voisinage des sclérites terminaux, chez $B$. risi (fig. 27 et 28 ) et chez $B$. beali. L'extrémité des microtriches est dirigée vers l'orifice vulvaire.

Le canal est plus long chez $B$. braueri que chez $B$. risi et $B$. beali, et surtout que chez $B$. monilicornis et $B$. seticornis ( $f i g .19$ à 22). Pour une espèce donnée, la longueur du canal du réceptacle de la femelle est donc en corrélation avec celle des filaments de l'épiprocte et du flagelle du paraprocte droit du mâle.

Le point d'aboutissement du canal dans le réceptacle est pourvu d'un sclérite dorsal et d'un sclérite ventral sur lesquels s'insèrent des muscles. Ces sclérites sont réunis sur les faces latérales par deux lames un peu sclérifiées dont l'élasticité doit avoir une action antagoniste de la contraction musculaire. Partout ailleurs, la paroi du réceptacle est mince, plissée, et se distend lors de l'arrivée du sperme.

\subsection{Accouplement}

Des $B$. braueri furent capturées près de la Garonne en amont de Toulouse le 2 février 1974. Les individus des deux sexes furent d'abord isolés plusieurs heures puis mis en présence. Aucun signal ne fut émis, contrairement à ce qu'a observé Rupprecht (1976) pour B. seticornis. Ces signaux ne sont donc pas nécessaires à l'accouplement mais il est probable qu'ils existent chez $B$. braueri comme chez les autres Plécoptères étudiés par Rupprecht (1968, 1969, 1972, 1976, 1977) 


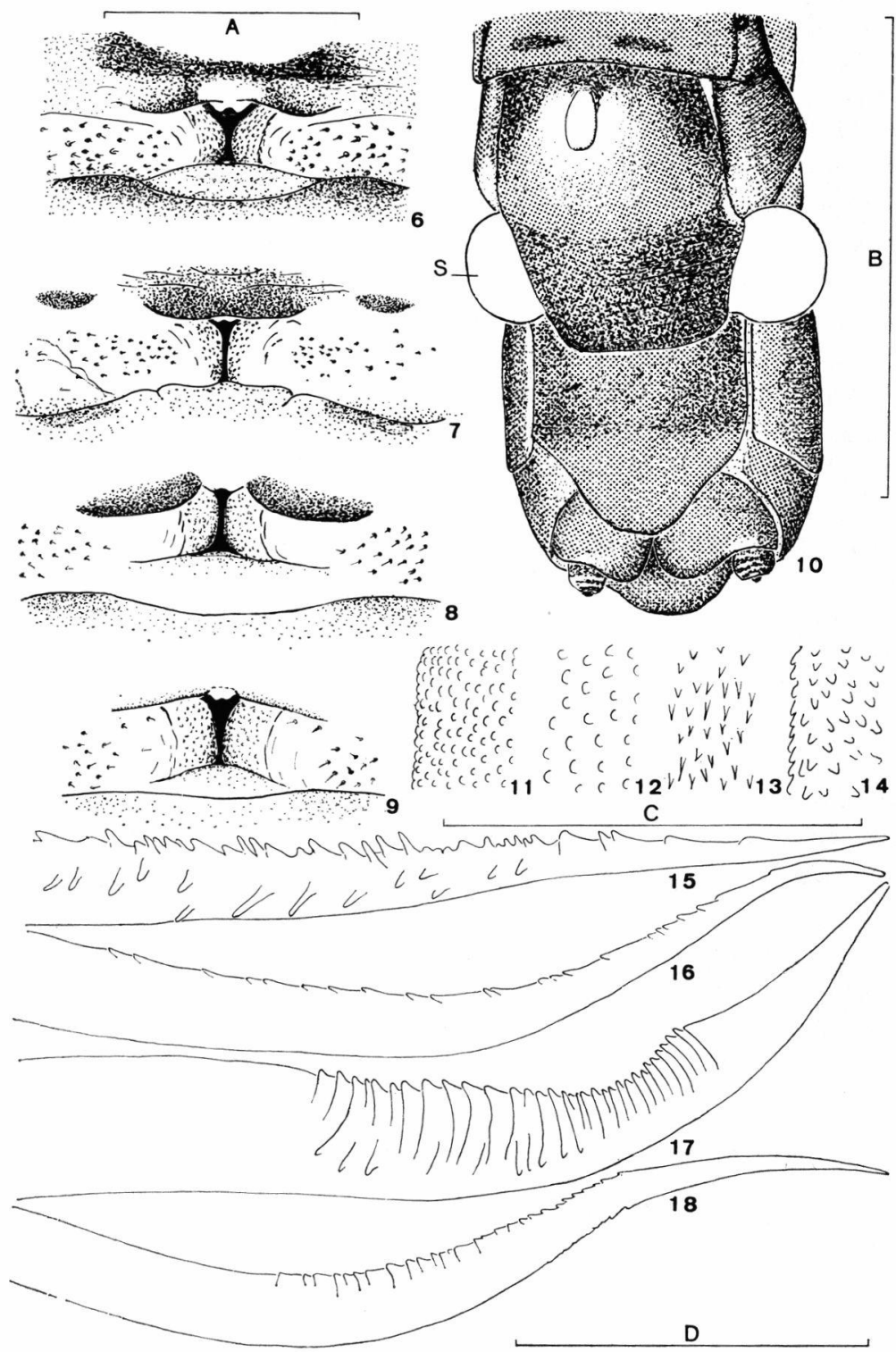

FIG. 6. - Orifice vulvaire de Brachyptera braueri. Fig. 7. - Id., B. monilicornis. FIG. 8. - Id., B. risi. Fig. 9. - Id., B. seticornis. Fig. 10. - Derniers segments abdominaux du mâle (en haut à gauche) et de la femelle de $B$. braueri lors de la sécrétion du spermatophore (S). FIG. 11. - Ornementation des bourrelets vulvaires de B. braueri. FIG. 12. - Id., B. monilicornis. FIG. 13. - Id., B. risi. FIG. 14. - Id., B. seticornis. FIg. 15. - Extrémité d'un filament de l'épiprocte de B. braueri. Fig. 16. - Id., B. monilicornis. FIg. 17. - Id., B. risi. FIG. 18. - Id., B. seticornis. Le trait $\mathrm{A}$ (fig. 6 à 9 ) correspond à $0,5 \mathrm{~mm}$, le trait $\mathrm{B}$ (fig. 10) à $2 \mathrm{~mm}$, le trait $\mathrm{C}$ (fig. 11 à 14$)$ à $0,1 \mathrm{~mm}$, le trait $\mathrm{D}$ (fig. 15 à 18$)$ à $0,05 \mathrm{~mm}$. 
et chez les Capnioneura, Tyrrhenoleuctra, Afroperlodes et Eoperla (observations personnelles).

La vue ne joue pas de rôle dans la recherche des femelles par les mâles. Ces derniers passent à un ou deux millimètres des femelles sans dévier leur marche. Dès le premier contact avec un autre indi-

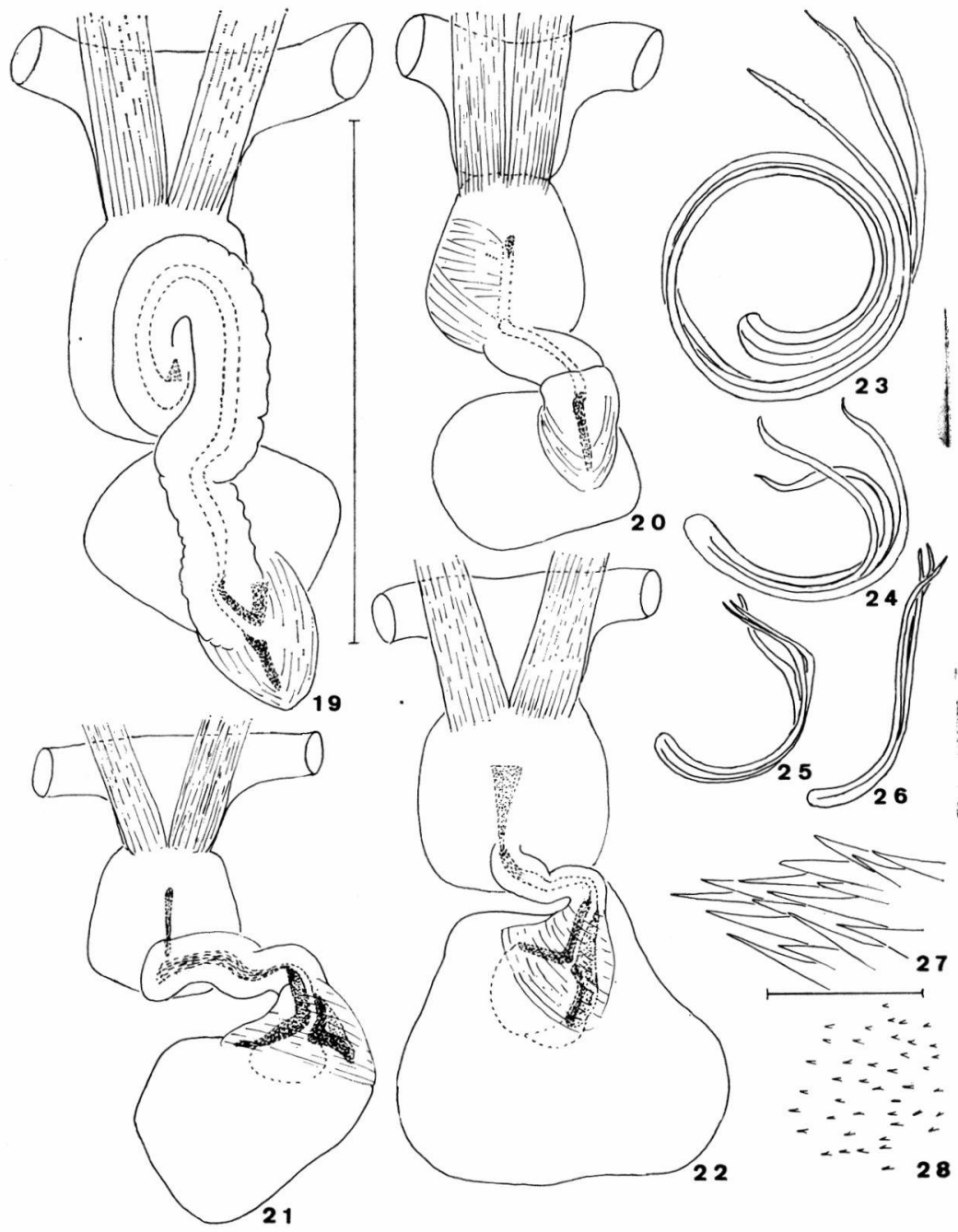

FIG. 19. - Vue dorsale de l'extrémité des oviductes, du vagin et du réceptacle séminal de Brachyptera braueri (femelle vierge). FIG. 20. - Id., B. monilicornis (à gauche, musculature propre du vagin). FIG. 21. - Id., B. risi. FIg. 22. - Id., B. seticornis. FIg. 23. - Filaments de l'épiprocte de B. braueri. Fig. 24. - Id., B. risi. FIg. 25. - Id., B. monilicornis. FIG. 26. - Id., B. seticornis. FIG. 27. Microtriches de l'entrée du canal du réceptacle de $B$. risi. FIg. 28. - Id., partie moyenne du canal. Le trait vertical ( $f$ ig. 19 à 26) correspond à $1 \mathrm{~mm}$, le trait horizontal (fig. 27 et 28 ) à $0,02 \mathrm{~mm}$. 
vidu, mâle ou femelle, le mâle en déplacement fait une tentative de chevauchement.

Pendant tout l'accouplement, le mâle se tient un peu en biais sur le dos de la femelle. Malgré la dissymétrie des paraproctes des $\mathrm{Bra}$ chyptera, un même mâle se place tantôt à droite tantôt à gauche, sans préférer l'un ou l'autre côté comme l'indique Brinck (1949, p. 119) pour divers Plécoptères de Suède.

Le mâle s'agrippe au prothorax et aux ailes de la femelle avec les pattes I, II et III situées du côté de sa partenaire. La femelle est aussi maintenue par la patte III du mâle située du côté opposé. Cette patte est glissée vers l'arrière entre les ailes et le dos du mâle et ses griffes s'accrochent au bord des ailes de la femelle. Cette curieuse attitude fut observée chez plusieurs couples de $B$. braueri et retrouvée chez des $B$. risi fixées in copula. Elle n'est pas signalée chez d'autres Plécoptères et peut être liée à la longue durée de l'accouplement.

L'accouplement comprend deux phases. La première dure d'une heure à plus de cinq heures (de 18 h 25 à 23 h 35 pour un couple). Le mâle introduit son épiprocte dans la vulve de la femelle. Le procès de l'épiprocte s'accroche au bord antérieur de la vulve. Le mâle contracte alors l'extrémité de l'abdomen, ce qui fait basculer l'épiprocte vers le haut et vers l'avant. Il est probable que ces contractions provoquent la dévagination des trois filaments. J'ai pu produire cette dévagination en pressant le bulbe de mâles fraîchement tués. Lorsque la pression cesse, les filaments s'enroulent à nouveau par élasticité. Mais au cours de l'accouplement, leurs aspérités subapicales (fig. 15 à 18) s'accrochent vraisemblablement à la paroi du canal du réceptacle séminal et empêchent une rétraction totale. Des contractions successives peuvent ainsi faire progresser les filaments dans le canal et, compte tenu de la corrélation entre les longueurs de ces organes (fig. 19 à 26), l'extrémité des filaments doit atteindre les sclérites du réceptacle.

Les contractions se succèdent tantôt à un rythme rapide d'un par seconde environ, tantôt à un rythme plus lent d'un toutes les cinq à dix secondes. Les appendices basaux des cerques du mâle frottent contre l'abdomen de la femelle. Les paraproctes ne jouent pas de rôle pendant toute cette période préliminaire.

Cette première phase rappelle celle que décrit Brinck (1962) pour Arcynopteryx compacta (Mc Lachlan, 1872). Dans les deux cas, l'épiprocte sert à stimuler la femelle mais ne participe pas au transfert du sperme. Il en est de même chez Afroperlodes (sub nom. Afroperla in Berthélemy et Laur 1976), dont la femelle absorbe ensuite activement le sperme déposé à l'extérieur à l'état liquide, comme chez Hydroperla crosbyi (Needham et Claassen, 1925), selon Stewart et Stark (1977).

La seconde phase de l'accouplement dure une quinzaine de minutes. L'abdomen du mâle est plus recourbé. Sa plaque sous-génitale, étroitement appliquée contre la face sternale de la femelle, masque les 
autres organes. D'après la position des deux partenaires, les paraproctes du mâle sont au niveau de la vulve. Le mâle émet le sperme et les sécrétions des glandes accessoires. L'ensemble déborde de part et d'autre de la plaque sous-génitale (fig. $10, \mathrm{~S}$ ). La couche superficielle durcit au contact de l'air. Le mâle se retire en laissant un spermatophore blanchâtre accroché à l'orifice génital de la femelle. Les bourrelets vulvaires sont rétractés, probablement depuis la phase de stimulation.

La femelle conserve pendant plusieurs heures le spermatophore qui se vide et se dessèche peu à peu. Il se détache lors d'un frottement de l'abdomen contre le substrat ou lors d'un deuxième accouplement. La même femelle peut en effet s'accoupler à plusieurs reprises en l'espace de quelques jours.

Dcs débris de spermatophore accrochés à la vulve ont été observés chez $B$. risi et $B$. seticomis. Ce mode de transfert du sperme paraît commun à toutes les Brachyptera, peut-être même à tous les Brachypterinae. Il permet de proposer une hypothèse sur le rôle des formations paraproctales. Le flagelle du paraprocte droit est parfois emboîté dans le prolongement membraneux du paraprocte gauche (fig. 5). L'ensemble est probablement introduit dans le vagin quand les bourrelets vulvaires sont rétractés. Lorsque les parois du spermatophore durcissent, la présence du flagelle ménage ainsi un canal interne pour l'écoulement du sperme.

La sécrétion d'un spermatophore externe est exceptionnelle chez les Plécoptères. D'ordinaire, le sperme est introduit dans le vagin grâce à l'épiprocte (Nemouridae, Capnia), aux paraproctes (Leuctra, Pachyleuctra), ou à un pénis ventral (nombreux Subulipalpia). Plus rarement, il est déposé à l'état liquide et absorbé activement par la femelle (Afroperlodes et Hydroperla).

Remarque. - Zwick (1973) figure une masse de sperme qui déborde de la vulve de deux Leuctridae américains, Zealeuctra et Moselia. Chez des Leuctra geniculata (Stephens, 1835) et $L$. madritensis Aubert, 1952, j'ai observé des masses semblables qui s'étaient coagulées à l'extérieur de l'orifice vulvaire et qui gardaient encore l'empreinte des paraproctes du mâle. La spermathèque de ces femelles était déjà pleine et, plutôt que d'un véritable spermatophore, il peut s'agir d'un bouchon spermatique ( sperme. Ces bouchons s'opposent, au moins momentanément, à une nouvelle insémination par un autre mâle.

\subsection{Période d'incubation}

Les femelles pondirent une semaine après les accouplements. C'est là un délai minimal car ces femelles n'étaient peut-être pas vierges au moment de leur capture. 
Les œufs, décrits par Brinck (1949), possèdent une couche gélatineuse externe plus dense que chez les autres Nemouroidea et les micropyles sont bien visibles.

Deux lots de trois cents œufs environ furent placés à la lumière du jour atténuée, à une température variant de 10 à $14^{\circ} \mathrm{C}$. Au bout de dix jours, un des lots fut transféré à l'obscurité, à une température de $10^{\circ} \mathrm{C}$. Le rythme des éclosions fut le même dans les deux cas.

Les membranes de l'œuf se déchirent longitudinalement, comme chez B. trifasciata (Pictet, 1832), selon Degrange (1957). Le ruptor ovi est formé par une dent sclérifiée située sur une coupole. Cette structure, figurée par Degrange (op. cit.), se retrouve chez tous les Plécoptères dont j'ai obtenu l'éclosion (Protonemura, Amphinemura, Nemoura, Capnopsis, Capnioneura, Tyrrhenoleuctra, Leuctra, Isoperla, Perlodes, Afroperlodes, Perla, Eoperla, Dinocras, Siphonoperla). Cette uniformité contraste avec la diversité rencontrée chez les Ephéméroptères (Degrange 1960) ou les Hétéroptères (Cobben 1968).

Les éclosions débutèrent trois semaines après la ponte et se poursuivirent pendant quatre semaines. Plus de la moitié des œufs sont éclos pendant les six premiers jours. Le pourcentage d'éclosion dépassa $95 \%$. D'après les observations réalisées chez d'autres Plécoptères, la durée d'incubation doit être supérieure d'une à deux semaines dans l'habitat naturel où les températures sont un peu plus basses.

Les œufs de $B$. braueri se développent sans diapause, contrairement à ceux de $B$. risi, selon Khoo (1968). Cette différence est probablement liée à l'habitat. $B$. braueri vit dans de grands cours d'eau où les variations thermiques estivales, moins brutales que dans les ruisseaux habités par $B$. risi, peuvent être plus aisément supportées par les jeunes larves.

\subsection{Premiers stades}

\subsubsection{STADE I (fig. 29 et 31 à 36)}

L'antenne comporte neuf articles (sept articles flagellaires), comme chez tous les Plécoptères paléarctiques. Une cicatrice marque l'emplacement du ruptor ovi sur le front. Les pièces buccales sont bien sclérifiées et fonctionnelles. L'extrémité de la lacinia est pourvue de denticulations ventrales et de soies dorsales.

Répartition des phanères sur chaque moitié du corps ${ }^{2}$

2. Les phanères des premiers stades sont assez peu nombreux pour être identifiés individuellement. Je me propose de les étudier chez le plus grand nombre possible d'espèces et de confronter la chétotaxie larvaire au schéma phylétique établi par Zwick (1973). 
a) Face dorsale

De courtes épines en avant du prothorax; sur celui-ci, six ou sept soies fines et une soie postérieure plus rigide.

Méso- et métathorax : deux épines antérieures; une forte soie tronquée latérale; trois soies fines; une soie postérieure plus rigide.

Abdomen : une (segments II à VII) ou deux (VIII à X, parfois quelques autres segments) fines soies près du bord antérieur; deux fortes soies postérieures (tous segments); une fine soie postérieure (VII à $\mathbf{X )}$.

b) Face ventrale

Sur chaque segment thoracique, une soie fine et de courtes épines en avant des hanches.

Abdomen : un pore circulaire (I et X) ; une courte épine antérieure (II) ; une soie postérieure fine (II à VIII) ou forte (IX); une forte soie postérieure latéroventrale ( $\mathrm{V}$ à $\mathrm{X}$ ).

Deux soies inégales sur chaque paraprocte.

Des cellules à chlorure coniformes (Wichard et Komnick 1973, Kapoor et Zachariah 1973 a, b) sont situées sur la face ventrale : cinq à sept paires sur le pro-, le méso- et le métathorax, une paire sur chacun des segments abdominaux $\mathrm{V}$ à $\mathrm{IX}$.

Chez les Plécoptères, la structure des cerques a varié à la fois pour le premier stade larvaire et pour le stade imaginal. Les cerques de l'adulte sont pluriarticulés et ceux du stade I sont formés de trois articles chez tous les Subulipalpia étudiés (Perlodes, voir fig. 38, Afroperlodes, Isoperla, Perla, Eoperla, Dinocras, Siphonoperla et d'après Khoo 1964, Isogenus, Diura et Chloroperla). Il en est de même chez quelques Nemouroidea (Capnia, Capniopsis et, d'après Khoo 1964, Taeniopteryx). C'est la disposition primitive, tout au moins pour les Arctoperlaria.

Les cerques sont réduits chez l'adulte et formés de quatre articles au stade I chez les Capnioneura, Tyrrhenoleuctra, Leuctra, Protonemura, Amphinemura, Nemoura et Nemurella. D'après le schéma phylétique de Zwick (1973), cette double transformation évolutive des cerques du stade I et de l'adulte s'est réalisée à trois reprises au moins (Capnioneura, souche des Leuctridae, souche des Nemouridae). Ce point est discuté plus loin (2.3.1.).

Les cerques de Brachyptera braueri présentent une structure intermédiaire. Au premier stade (fig. 29, 35 et 36), une couronne incomplète de fortes soies est insérée au milieu du premier des trois articles. Chez la femelle adulte, les cerques présentent des traces de segmentation (fig. 10 et figures de Kis 1974).

Khoo (1964) indique que le stade I de Brachyptera risi possède des 


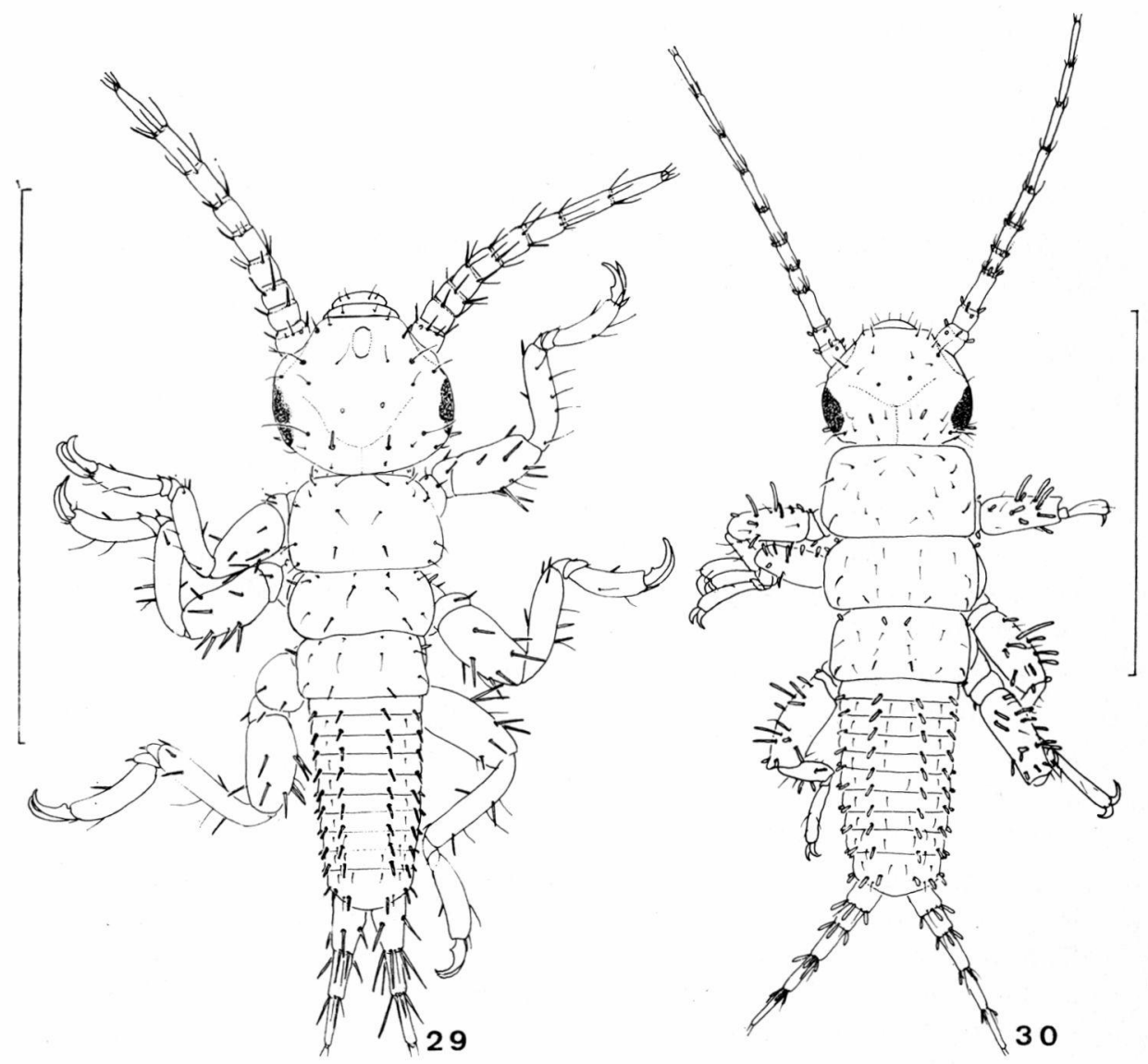

Fig. 29. - Face dorsale du premier stade larvaire de Brachyptera braueri. Fig. 30. Id., troisième stade. Les traits de gauche (fig. 29) et de droite (fig. 30) correspondent à $0,5 \mathrm{~mm}$.

cerques de quatre articles. Il s'agit probablement d'un dénombrement basé sur les couronnes de soies et non sur la présence des membranes articulaires (voir ci-dessous une même discordance pour le stade II de Perlodes microcephalus).

\subsubsection{STADE II}

Les fortes soies sont plus courtes et plus dilatées à l'extrémité. Les fines soies dorsales antérieures sont plus fréquentes sur les premiers segments abdominaux. Le bord postérieur du dixième segment abdominal porte une paire supplémentaire de fortes soies. Les cellules à chlorure sont plus nombreuses et irrégulièrement distribuées sur la face ventrale du thorax et des premiers segments abdominaux. Les cerques comprennent quatre articles complètement séparés (fig. 37). 

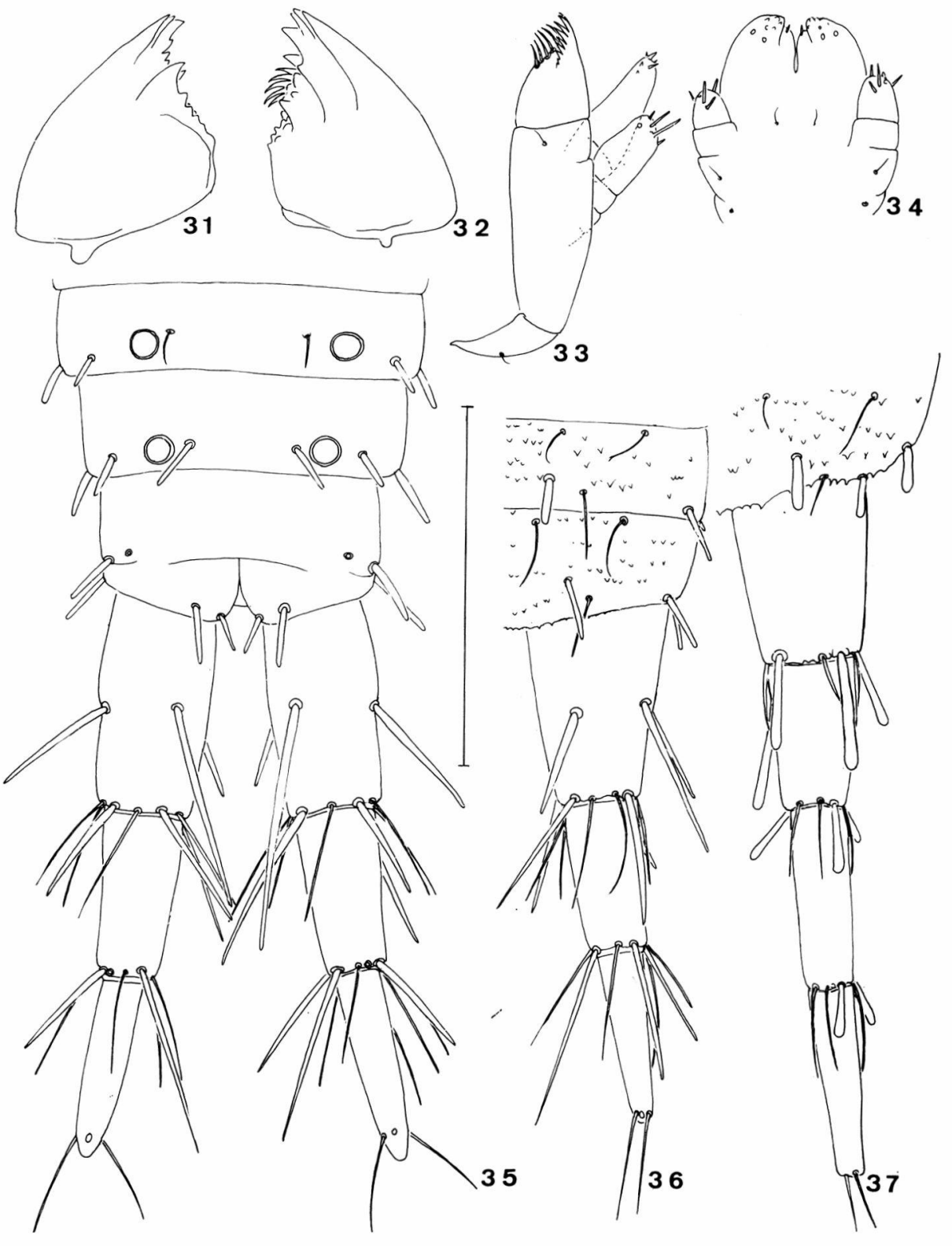

Fig. 31. - Premier stade larvaire de Brachyptera braueri, face ventrale de la mandibule droite. FIG. 32. - Id., mandibule gauche. FIG. 33. - Id., maxille. Fig. 34. - Id., labium. Fig. 35. - Id., extrémité postérieure. Fig. 36. - Id., face dorsale de la moitié droite de l'extrémité postérieure. - FIG. 37. - Deuxième stade larvaire de $B$. braueri, face dorsale de la moitié droite de l'extrémité postérieure. Le trait vertical correspond à $0,1 \mathrm{~mm}$. 


\subsubsection{STAdE III}

Le flagelle de l'antenne est formé de huit articles (fig. 30). La forme des fortes soies se rapproche de celle des larves âgées (Berthélemy et Laur 1976, fig. $3 \mathrm{~b}$ ). Le neuvième segment abdominal porte une paire supplémentaire de fortes soies. Les cerques comportent cinq articles.

Sur la face ventrale, le segment IX n'est pas prolongé en arrière et le segment $\mathrm{X}$ n'est pas rétréci au milieu. Les caractères des larves âgées apparaissent donc à un stade ultérieur.

D'après Khoo (1964), chez le stade III de $B$. risi, le flagelle de l'antenne est formé de treize articles et les cerques de dix articles. Cet accroissement plus rapide compense peut-être la diapause embryonnaire.

\section{2. - PERLODES MICROCEPHALUS (PICTET, 1833)}

\subsection{Accouplement}

Les données suivantes complètent la description de Brinck (1956) pour Perlodes dispar (Rambur, 1842).

Comme chez Brachyptera braueri, les appels sexuels ne sont pas nécessaires pour que la femelle accepte le mâle. Les deux derniers segments abdominaux du mâle sont fortement incurvés vers le haut et le dernier est ainsi perpendiculaire aux segments moyens. Par des mouvements latéraux de l'abdomen, le mâle frotte le ventre de la femelle avec les fortes soies dorsales de la base des cerques. La femelle semble réagir par une contraction des deux derniers segments qui fait saillir la plaque sous-génitale. Le mâle accroche cette plaque avec ses paraproctes. Comme l'indique Brinck (1956), et contrairement à l'hypothèse de Stewart et Stark (1977), le pénis est ensuite complètement introduit dans le vagin. Les paraproctes du mâle sont alors écartés de la plaque sous-génitale. A aucun moment le sperme n'est répandu à l'extérieur.

\subsection{Période d'incubation}

L'œuf a été décrit par Percival et Whitehead (1928, sub nom. Perlodes mortoni), Hynes (1941, id.), Berthélemy (1964), Schwarz (1970) et Kis (1974).

D'après Percival et Whitehead, la durée d'incubation est de 91 jours à $15,5^{\circ} \mathrm{C}$ environ. Khoo (1964) indique des durées de 33 à 54 jours $\left(13\right.$ à $\left.23^{\circ} \mathrm{C}\right), 78$ à 110 jours $\left(7\right.$ à $13^{\circ} \mathrm{C}$, simulant les températures naturelles) et de plus de 194 jours ( 1 à $7^{\circ} \mathrm{C}$ ). Schwarz (1970) note des durées de 56 à 70 jours selon la température. 
Mes premiers résultats confirmèrent les précédents. Une ponte du 8 avril fut placée à une température de 15 à $18^{\circ} \mathrm{C}$ en lumière du jour atténuée jusqu'au 20 mai, puis à $15^{\circ} \mathrm{C}$ à l'obscurité. Les éclosions commencèrent début juin (durée minimale d'incubation, 58 jours) et se poursuivirent jusqu'à fin septembre (durée maximale, 167 jours). Quatre autres pontes furent placées après le 20 mai dans des conditions un peu différentes $\left(10^{\circ} \mathrm{C}\right.$ à l'obscurité, 16 à $21^{\circ} \mathrm{C}$ à la lumière du jour atténuée). Ces températures sont les unes un peu inférieures, les autres un peu supérieures à celles de la station d'origine, le Lez à $430 \mathrm{~m}$ (voir Berthélemy 1966). Plus de 1500 éclosions furent obtenues entre début juin et fin septembre, sans différences nettes selon les conditions expérimentales.

Deux autres pontes des mêmes femelles, obtenues le 24 et le 26 avril, furent placées après le 20 mai à une température variant de 11 à $14^{\circ} \mathrm{C}$, à la lumière du jour atténuée. Ces conditions correspondent à celles que rencontre $P$. microcephalus dans certains cours d'eau. Plus de 900 œufs sont éclos entre le 15 juillet et le 15 octobre, avec un net maximum en août. Ce résultat s'accordait bien avec toutes les données précédentes. Mais les œufs non éclos furent conservés dans les mêmes conditions, ce qui n'avait pas été le cas pour les autres pontes, ni, vraisemblablement, pour les pontes étudiées par les autres auteurs. Vingt-cinq œufs sont alors éclos entre le 20 avril et la fin juillet de l'année suivante, après une interruption de plus de cinq mois.

Les œufs d'une même ponte, placés dans un seul récipient, peuvent donc soit se développer en trois, quatre ou cinq mois, soit éclore au bout d'un an. Cette diapause se situe au début du développement embryonnaire, et non à la fin comme chez Isoperla goertzi Illies, 1952, selon Schwarz (1970).

Le mode d'éclosion est voisin de celui de Dinocras cephalotes (Curtis, 1827), décrit par Degrange (1957), mais le chorion se fissure ici selon une ligne de rupture déjà visible sur l'œuf vierge.

\subsection{Premiers stades}

\subsubsection{Stade I (fig. 38)}

Les pièces buccales sont peu sclérifiées et non fonctionnelles ( $f$ ig. 39 à 41). Les larves au premier stade muent sans s'être alimentées. Il en est de même chez les Isoperla, Afroperlodes, Perla, Eoperla, Dinocras, Siphonoperla et selon Khoo (1964), Isogenus, Diura et Chloroperla.

On retrouve donc, pour les pièces buccales des Subulipalpia, le parallélisme entre le stade I et l'adulte signalé pour les cerques des Nemouroidea (voir ci-dessus 1.4.1.). Dans les deux cas, le développement embryonnaire et la mue imaginale ont été modifiés simultanément, ici par réduction de la sclérification des pièces buccales. Cette 
double évolution est probablement due aux effets pléiotropiques de certaines mutations. Ces effets ne se manifestent pas pendant la période intermédiaire, du stade II au stade préimaginal, peut-être sous l'influence d'une hormone juvénile. Une origine indépendante de ces caractères est moins vraisemblable. On voit mal, en effet, quelle transformation du mode de vie pourrait être liée à la fois à une augmentation du nombre des articles des cerques à la naissance et à une réduction des cerques chez l'adulte.

\section{Répartition des phanères sur chaque moitié du corps}

a) Face dorsale

De petites épines en avant du prothorax; sur celui-ci, huit à neuf

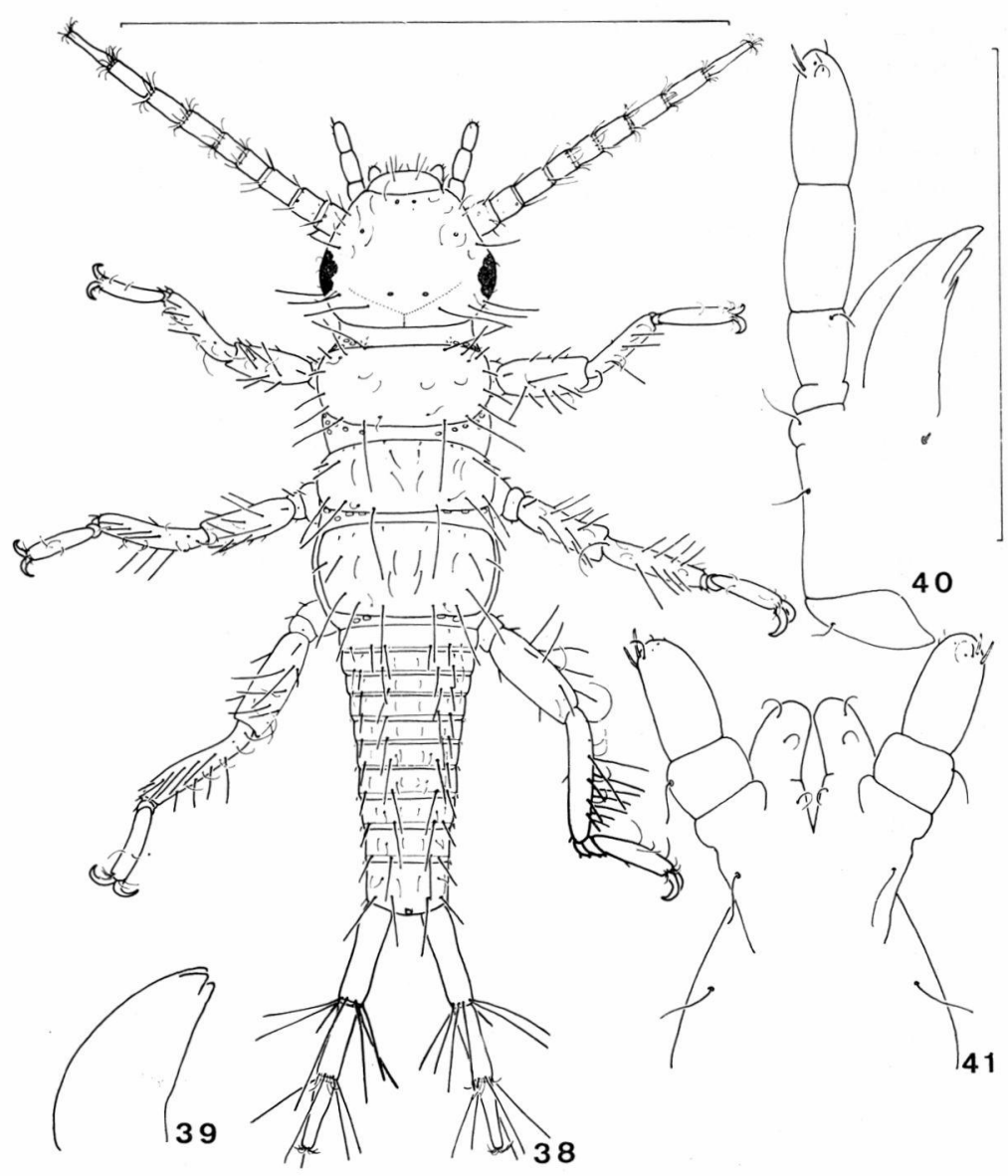

Fig. 38. - Face dorsale du premier stade larvaire de Perlodes microcephalus.

FIG. 39. - Face ventrale de la mandibule droite. FIG. 40. - Id., maxille droite. FIG. 41. - Id., labium. Le trait horizontal (fig. 38) correspond à $1 \mathrm{~mm}$, le trait vertical (fig. 39 à 41 ) à $0,2 \mathrm{~mm}$. 
fortes soies marginales, deux fines soies sur le disque, un poil antérieur et un poil postérieur à grande cupule d'insertion.

Méso- et métathorax: trois petites épines antérieures; une forte soie antérieure ; quatre fortes soies marginales ; trois, parfois quatre soies de taille moyenne sur le disque ; un poil postérieur.

Abdomen : une épine antérieure (segments II à IV, souvent aussi I) ; une (II à IV) ou deux (V à X) fines soies insérées vers le milieu des

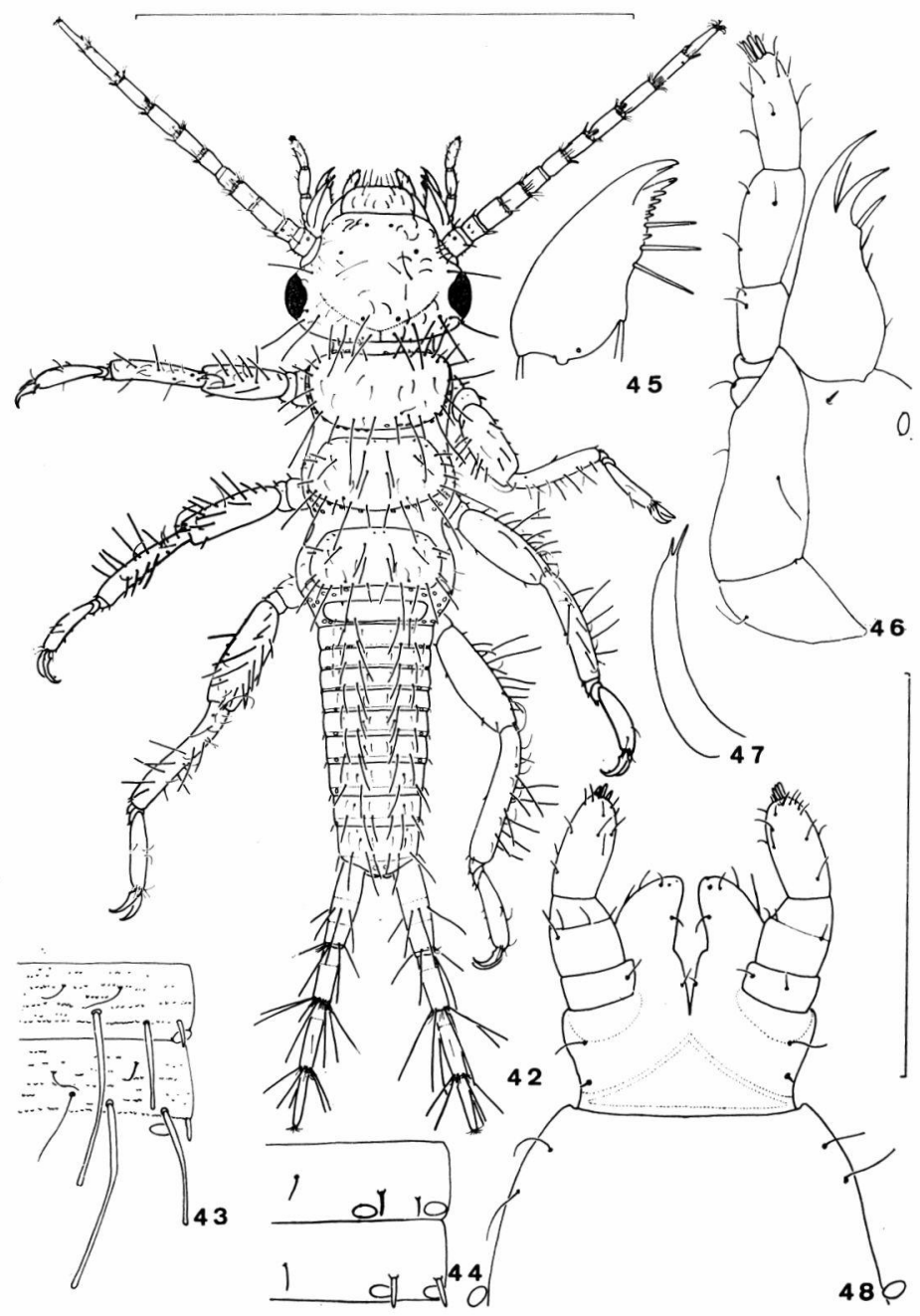

Fig. 42. - Face dorsale du deuxième stade larvaire de Perlodes microcephalus. FIG. 43. - Face dorsale de la moitié droite des segments abdominaux V et VI. FIG. 44. - Face ventrale de la moitié gauche des segments abdominaux $\mathrm{V}$ et VI. FIG. 45. - Face ventrale de la mandibule droite. FIG. 46. - Id., maxille droite, galéa non figurée. Fig. 47. - Id., galéa. FIG. 48. - Id., labium. Le trait horizontal (fig. 42) correspond à $1 \mathrm{~mm}$, le trait vertical (fig. 43 à 48 ) à $0,2 \mathrm{~mm}$. 
segments; un poil situé entre les précédentes (VI à X); deux fortes soies postérieures (tous segments); une fine soie latérale postéricure (I à III).

\section{b) Face ventrale}

Sui: chaque segment thoracique, de courtes épines et deux fines soies en avant des hanches.

Abdomen : un poil sensoriel (I) ; une épine antérieure (II, III, IV, IX et $\mathrm{X}$ ) ; une fine soie insérée vers le milieu des segments (II à VII) ; une (II et III), deux (IV à VIII) ou trois (IX et X) soies postérieures, fines sur les cinq ou six premiers segments et plus fortes sur les suivants.

Une fine soie sur chaque paraprocte.

Des cellules à chlorure coniformes sont situées en avant du pronotum (trois à cinq cellules), en arrière du pronotum (cinq à sept), en arrière du mésonotum (trois à quatre), en arrière du métanotum (deux à trois), sur la face ventrale du prothorax (trois à quatre), du mésothorax (quatre à sept) et du métathorax (quatre à cinq). Tous les nombres précédents se rapportent à une moitié du corps.

Sur chaque face latérale de l'abdomen se trouve une (segments I et IX) ou deux (II à VIII) cellules. Le dernier segment porte une cellule impaire dorsale.

Les cerques ont des couronnes de soies fortes (articles I et II) et fines (II et III).

\subsubsection{Stade II (fig. 42)}

Le flagelle de l'antenne comporte neuf articles, dont les deux premiers sont parfois incomplètement séparés. Cet accroissement du nombre des articles au cours de la première mue est exceptionnel chez les Subulipalpia. Dans les genres déjà cités (2.3.1.), le stade II possède un flagelle de sept articles, comme le stade I. La croissance de $P$. microcephalus est particulièrement rapide puisqu'il atteint en un an une taille supérieure à celle des Perlidae âgés de deux ans.

Les pièces buccales sont bien sclérifiées et fonctionnelles (fig. 45 à 48). L'intestin antérieur et l'intestin postérieur sont revêtus de denticulations chitineuses.

Les sclérites dorsaux et latéraux du méso- et du métathorax sont pigmentés, ainsi que, à un degré moindre, les antennes, le labre, le clypéus, les hanches, les trochanters et les fémurs.

Des soies supplémentaires sont apparues en divers points. Le pronotum porte de chaque côté douze à quinze fortes soies marginales et quatre ou cinq fines soies sur le disque. 
Les trois soies de taille moyenne du méso- et du métanotum sont remplacées par de fortes soies et trois nouvelles soies plus fines se sont formées. Les fortes soies marginales sont au nombre de six à huit.

Une paire supplémentaire de fortes soies dorsales est insérée au bord postérieur des segments abdominaux IX à $X$, de même que, parfois, une nouvelle soie en position asymétrique sur les segments VII ou. VIII.

Ventralement, les segments thoraciques portent quelques fines soies irrégulièrement disposées. Le bord postérieur des segments abdominaux est muni d'une (segment VIII et X) ou deux (IX, parfois X) paires supplémentaires de fortes soies. Une paire de fines soies est apparue au milieu des segments VIII à $X$.

Les nombre des cellules à chlorure a augmenté aux emplacements qu'elles occupent déjà au stade I : pour chaque moitié du corps, quatorze à seize sur tout le pourtour du pronotum, six à huit en arrière du mésonotum, cinq à six en arrière du métanotum, trois à cinq sur les côtés des segments abdominaux I à VIII, une à trois sur les côtés du segment IX, souvent deux en arrière du dernier segment.

En outre, il en est apparu sur la face ventrale de la tête (fig. 46 et 48) et des fémurs (deux, plus rarement une ou trois cellules), ainsi que sur les paraproctes (une cellule).

Les cerques sont formés de quatre articles. Une couronne de fortes soies est insérée au milieu du premier, ce qui explique probablement que Khoo (1964) et Schwarz (1970) indiquent l'existence de cinq articles. L'article I présente deux stries transversales, les articles II et III unc strie chacun.

\section{REMERCIEMENTS}

Je remercie très vivement M. M. Thibault (I.N.R.A., Rennes) qui m'a communiqué une partie des Brachyptera monilicornis étudiées ici, ainsi que le $\mathrm{D}^{r} \mathrm{P} . \mathrm{P}$. Harper (Montréal) qui m'a procuré la thèse de S. G. Khoo (1964).

\section{TRAVAUX CITÉS}

Berthélemy (C.). 1964. - Intérêt taxonomique des cufs chez les Perlodes européens (Plécoptères). Bull. Soc. Hist. nat. Toulouse, 99 (3/4) : 529-537.

Berthélemy (C.). 1966. - Recherches écologiques et biogéographiques sur les Plécoptères et Coléoptères d'eau courante (Hydraena et Elminthidae) des Pyrénées. Annls Limnol., 2 (2) : 227-458.

Berthélemy (C.). 1971. - Plécoptères de Grèce centrale et d'Eubée. Biologia GalloHellenica (1970), 3 (1) : 23-56.

Berthélemy (C.) et Laur (C.). 1976. - Plécoptères et Coléoptères aquatiques du Lot (Massif Central français). Annls Limnol. (1975), 11 (3) : 263-285. 
BRINCK (P.). 1949. - Studies on Swedish Stoneflies (Plecoptera). Opusc. ent., Suppl. $11: 1-250$.

BriNck (P.). 1952. - Bäcksländor. Plecoptera. Svensk Insektfauna, 15: 128 p. Stockholm.

BRINCK (P.). 1956. - Reproductive system and mating in Plecoptera. Opusc. ent., $21: 57-127$.

BRINCK (P.). 1962. - Begattungsorgane und Spermaübertragung bei den Isogeninen Perlodiden. Verh. XI. internat. Kongr. Ent. Wien 1960, 3 : 267-269.

Cobben (R. H.). 1968. - Evolutionary trends in Heteroptera. Part I : Eggs, architecture of the shell, gross embryology and eclosion. 475 p. Centre for agricultural publishing and documentation, Wageningen.

Degrange (Ch.). 1957. - L'œuf et le mode d'éclosion de quelques Plécoptères. Trav. Lab. Hydrobiol. Piscic. Univ. Grenoble, 48/49: 37-49.

Degrange (Ch.). 1960. - Recherches sur la reproduction des Ephéméroptères. Trav. Lab. Hydrobiol. Piscic. Univ. Grenoble, 50/51 : 7-193.

Despax (R.). 1951. - Faune de France, 55 : Plécoptères. 280 p., P. Lechevalier, Paris.

HoffmanN (J.). 1960. - Faune des Plécoptères du Grand-Duché de Luxembourg. Archs Inst. Gr.-Duc. Luxembourg, 27 : 121-208.

Hynes (H. B. N.). 1941. - The taxonomy and ecology of the nymphs of British Plecoptera with notes on the adults and eggs. Trans. r. ent. Soc. London, $91: 459-557$.

InLIEs (J.). 1955. - Steinfliegen oder Plecoptera. Die Tierwelt Deutschlands und der angrenzenden Meeresteile, $43: 150$ p. G. Fischer, Jena.

Kapoor (N. N.) et Zachariah (K.). 1973 a. - Presence of specialized cellular complexes in the tracheal gills of Stonefly nymph, Paragnetina media (Walker). Experientia, $29: 848-850$.

KapooR (N. N.) et Zachariah (K.). 1973 b. - A study of specialized cells of the tracheal gills of Paragnetina media (Plecoptera). Can. J. Zool., 51 (9) : 983986.

Kно0 (S. G.). 1964. - Studies on the biology of Stoncflies. Unpublished thesis, University of Liverpool, $161 \mathrm{p}$.

KHоo (S. G.). 1968. - Experimental studies on diapause in Stoneflies. III. Eggs of Brachyptera risi (Morton). Proc. r. ent. Soc. London (A), 43 (10/12) : 141-146.

KIS (B.). 1974. - Plecoptera. Fauna Republicii Socialiste România. Insecta, 8 (7) : 273 p. Bucarest.

KLAPÁLEK (F.). 1896. - Uber die Geschlechstheile der Plecopteren, mit besonderer Rücksicht auf die Morphologie der Genitalanhänge. Sber. K. Akad. Wiss. Wien, math.-naturw. Kl., 105:683-738.

Percival (E.) et Whitehead (H.). 1928. - Observation on the ova and oviposition of certain Ephemeroptera and Plecoptera. Proc. Leeds phil. lit. Soc. scient. Sect., I : 271-288.

Rupprecht (R.). 1968. - Das Trommeln der Plecopteren. Z. vergl. Physiol., 59 (1) : 38-71.

RuPPRECHT (R.). 1969. - Zur Artspezifität der Trommelsignale der Plecopteren (Insecta). Oikos, $20: 26-33$.

RuPPRECHT (R.). 1972. - Dialektbildung bei den Trommelsignalen von Diura (Plecoptera). Oikos, $23: 410-412$.

RUPPRECHT (R.). 1976. - Struktur und Funktion der Bauchblase und des Hammers von Plecopteren. Zool. Jb. Anat., 95 : 9-80.

RuPPRECHT (R.). 1977. - Nachweis von Trommelsignalen bei einem europäischen Vertreter der Steinfliegen-Familie Leuctridae (Plecoptera). Ent. Germ., 3 (4) : 333-336.

ScHWARZ (P.). 1970. - Autökologische Untersuchungen zum Lebenzyklus von Setipalpia-Arten (Plecoptera). Arch. Hydrobiol., 67, I : 103-140 et II : 141-172. 
Stewart (R. W.) et StaRK (B. P.). 1977. - Reproductive system and mating of Hydroperla crosbyi: a newly discovered method of sperm transfer in Insecta. Oikos, $28: 84-89$.

WichaRd (W.) et KoMnick (H.). 1973. - Feinstruktureller und histochemischer Nachweis von Chloridzellen bei Steinfliegenlarven. I. Die coniformen Chloridzellen. Cytobiologie, 7 (3) : 297-314.

Zwick (P.). 1973. - Insecta : Plecoptera. Phylogenetisches System und Katalog. Das Tierreich, 94 : XxxII + 465 p. W. de Gruyter, Berlin. 